\title{
Familiarity and attention: Does what we know affect what we notice?
}

\author{
JOHN CHRISTIE and RAYMOND KLEIN \\ Dalhousie University, Halifax, Nova Scotia, Canada
}

\begin{abstract}
Previous work on the object and word superiority effects has demonstrated that activation from stored representations can facilitate identification of items in a visual display. We predicted that activation of this sort might exogenously attract visual attention toward items that have stored representations. To test this prediction, we presented a familiar (word) and an unfamiliar (nonword) item simultaneously at unpredictable locations, and after varying delays, moved one of the stimuli. In accord with our prediction, at the shortest intervals subjects were more efficient at discriminating motion of the familiar item. Control data demonstrated that this advantage was due to a competitive interaction and not to the familiarity of the items per se.
\end{abstract}

In the recent history of cognitive psychology, investigators have been interested in whether familiarity (i.e., whether a subject has a stored representation of an item) has any effect on the allocation of visual-spatial attention (see, e.g., Flowers \& Lohr, 1985; Flowers, Polansky, \& Kerl, 1981; Johnston, Hawley \& Farnham, 1993; Johnston, Hawley, Plewe, Elliott, \& Dewitt, 1990; Krueger, 1970; Reicher, Snyder, \& Richards, 1976). In this short report we will outline important methodological conditions that must be considered when one addresses this question and will present an experiment that satisfies these conditions.

How should one test whether relative familiarity exerts exogenous control over visuospatial attention? Since it is well established that perception of attended objects is enhanced, relative to unattended ones (Hawkins, Hillyard, Luck, Mouloua, Downing, \& Woodward, 1989; Posner, Snyder, \& Davidson, 1980), the processing of items that attract attention should be faster and/or more accurate than the processing of items that do not. The ubiquitous word and object superiority effects, which reveal that the identity and component parts of familiar/meaningful items are more accurately reported than those of unfamiliar/meaningless items, might be interpreted as support for the hypothesis that attention will be allocated to familiar items. These superiority effects strongly imply contributions from stored representations that are more elaborate for familiar than for unfamiliar stimuli (McClelland \& Rumelhart, 1981; Mewhort \& Johns, 1988). But, because these tasks assess the report of information that is present both in the stimulus and in the stored representation, the possible effect upon, and contribution from, spatial attention cannot be inde-

The research reported here was supported by a grant from the Natural Sciences and Engineering Research Council to R. Klein. Thanks to Bill Johnston and an anonymous reviewer for their insightful comments. Special thanks to Tracy Taylor for her help in enhancing the clarity of this manuscript. Correspondence should be addressed to J. Christie, Psychology Department, Dalhousie University, Halifax, N.S., Canada B3H 4J1 (e-mail: in\%"jc@ac.dal.ca"). pendently verified. Thus, one condition that must be satisfied in order to assess the locus of attention with respect to item familiarity is that the reported attribute of the familiar and unfamiliar items (which is used to index the locus of attention) should not be available from the stored representation (orthogonal probe dimension). This condition was first satisfied by Earhard (1968), who demonstrated that the detection of a thinner letter is unaffected by letter string familiarity. This important finding cannot speak to the attentional issue, because in Earhard's study, only one item was presented on each trial and the subject knew where it would occur. Therefore, each item, whether familiar or unfamiliar, was already attended. Thus, a second condition that must be satisfied to test the hypothesis is that at least one familiar and one unfamiliar item must be displayed at the same time in unpredictable locations (unpredictable target location).

No studies that we are aware of have simultaneously satisfied both of these conditions. To do so is the purpose of this study. Our design involved the simultaneous presentation of familiar (word) and unfamiliar (nonword) stimuli in unpredictable locations. One of the stimuli moved either up or down and the subject was asked to discriminate the direction of the movement, thereby satisfying our criterion that the probed attribute of the array item not be available from stored representations.

When subjects are assessed according to our criteria of unpredictable target location and orthogonal probe dimension, will attention be influenced by familiarity, and if so, will familiar or unfamiliar items attract it? In models of pattern perception and object recognition, it is usually assumed that the system responsible for the encoding of a visual scene or display, which we will refer to as the visual buffer (see Kosslyn, 1980), is richly interconnected with a system of stored representations (see, e.g., Farah, 1990; Kahneman, 1973). Indeed, feedback from representations of words (familiarity-based representations) to lower levels is now widely accepted as the mechanism for the word superiority effect (McClelland \& Rumelhart, 1981). We 
propose that familiar items will exert a stronger pull on attention than unfamiliar ones will, because the region in the visual buffer occupied by a familiar item will become more highly activated (than the region occupied by an unfamiliar one) because of connections from the stored representations. This exogenous influence on attention should occur early on in the processing of a visual display. At later stages of processing, however, this initial effect might decline or even reverse, because, as the content of the familiar location is verified, attention may be strategically (endogenously) reallocated toward novel items to assist in their identification (see Klein, Kingstone, \& Pontefract, 1992, for a treatment of the exogenous/endogenous distinction).

We recognize fully that our proposal appears to contradict some recent ideas on the relationship between novelty and attention (see, e.g., Johnston et al., 1990; Johnston et al., 1993; Loftus \& Mackworth, 1978). In our discussion, we will demonstrate that these ideas are either not relevant to the present question and/or do not meet the criteria we have set forth for addressing it.

\section{METHOD}

\section{Subjects}

There were 30 subjects with normal or corrected-to-normal vision. These consisted of 18 females and 12 males between the ages of 16 and 34 .

\section{Apparatus and Stimuli}

Stimuli were presented, and data were recorded, by an MBD 11/23 computer. The stimuli were displayed on a Tektronix 604 oscilloscope with fast phosphor and consisted of the words and nonwords shown in the Appendix. An Eyetrac 210 was used to monitor vertical and horizontal eye position for all tasks. Responses were made on a two-key switch pad with the index and middle fingers of the right hand, and individual trials began when the subject pressed a thumb switch held in the left hand. Each stimulus display consisted of a pair of words presented horizontally in a diagonal arrangement (bottom left and top right, or the top left and bottom right corners). Twenty-four highly familiar five-letter words were selected from Gernsbacher (1984, Appendix, Experiments 3-4). The Gernsbacher corpus was selected, because the word familiarity was derived through experimental research rather than written frequency methods. For each word stimulus a corresponding unfamiliar and unpronounceable letter string was constructed by scrambling the word's letters. Unpronounceable strings were used to ensure that these un- familiar strings would be unlikely to contact representations in the orthographic lexicon. The letter strings subtended a visual angle $2.77^{\circ}$ horizontally and $0.55^{\circ}$ vertically with $4.61^{\circ}$ of separation between the centers. The target event was a vertical shift of one of the letter strings, which was accomplished by redrawing it displaced by $\pm 0.18^{\circ}$.

\section{Task, Procedure, and Design}

All subjects were instructed about the task and stimuli and then performed a calibration procedure that permitted the experimental program to reject trials when the subject failed to maintain fixation for the duration of a trial. The room was darkened and the subject's comfort in the chinrest was verified before the commencement of the experimental session. After the subject initiated a trial, there was a 300-msec delay until two letter strings were presented. After 100 , 200 , or $400 \mathrm{msec}$, one of the strings appeared to move either up or down. The subject was required to indicate as rapidly and as accurately as possible whether the change was an upward or a downward movement by pressing the response keys with the middle and index fingers, respectively. Upward and downward movements occurred equally often across all conditions.

The subject first received a practice block that consisted of the 24 trials containing simple strings of $x \mathrm{~s}$ and $y \mathrm{~s}$. This was followed by one test block consisting of 192 trials. Experimental trials consisted of one word (W) and one nonword (N). Trials in which the word was target are labeled WN, and trials in which the nonword was target are labeled NW. Control trials, in which both of the stimuli were of the same category, either familiar (WW) or unfamiliar (NN), were intermixed with the experimental trials. All factors were counterbalanced so that on any trial a subject was equally likely to receive nonword or word stimuli in any of the four stimulus locations; this was constrained only by the locations' being diagonally opposite.

To test the hypothesis that familiar items attract attention, we compared reaction times (RTs) in the WN and NW conditions. It was predicted that when the target motion occurred soon after the stimulus array was presented performance on WN trial movement discriminations would be superior to that in NW trials, and that this difference might dissipate with increasing stimulus onset asynchrony (SOA). The purpose of the control conditions was to determine whether any effects on the perception of movement were due merely to the nature (familiarity) of the stimuli. On the basis of Earhard's (1968) findings, it was expected that $\mathrm{WW}=\mathrm{NN}$.

\section{RESULTS}

A single-pass outlier check was run on the data from each of 29 of the subjects. ${ }^{1}$ Scores that deviated more than 3 standard deviations from the mean RT in each condition

Table 1

Mean Reaction Time (RT, in Milliseconds), Percentage Correct, and Efficiency Measures for All Conditions

\begin{tabular}{|c|c|c|c|c|c|c|c|c|c|}
\hline \multirow[b]{3}{*}{ Condition } & \multicolumn{9}{|c|}{ SOA } \\
\hline & \multicolumn{3}{|c|}{100} & \multicolumn{3}{|c|}{200} & \multicolumn{3}{|c|}{300} \\
\hline & RT & $\%$ Corr. & Eff. & $\mathrm{RT}$ & $\%$ Corr. & Eff. & RT & $\%$ Corr. & Eff. \\
\hline \multicolumn{10}{|c|}{ Experimental } \\
\hline $\mathrm{WN}$ & 478 & 91.6 & 527 & 466 & 94.9 & 492 & 463 & 92.5 & 501 \\
\hline NW & 499 & 92.2 & 545 & 469 & 92.9 & 512 & 464 & 93.3 & 502 \\
\hline \multicolumn{10}{|l|}{ Control } \\
\hline WW & 486 & 90.2 & 549 & 468 & 93.3 & 503 & 454 & 92.3 & 494 \\
\hline NN & 498 & 94.4 & 533 & 470 & 93.9 & 506 & 460 & 94.2 & 488 \\
\hline
\end{tabular}

Note-SOA, stimulus onset asynchrony; W, word; N, nonword. WN: the word was the target. NW: the nonword was the target. In control trials, both stimuli were of the same category, either familiar (WW) or unfamiliar (NN). 


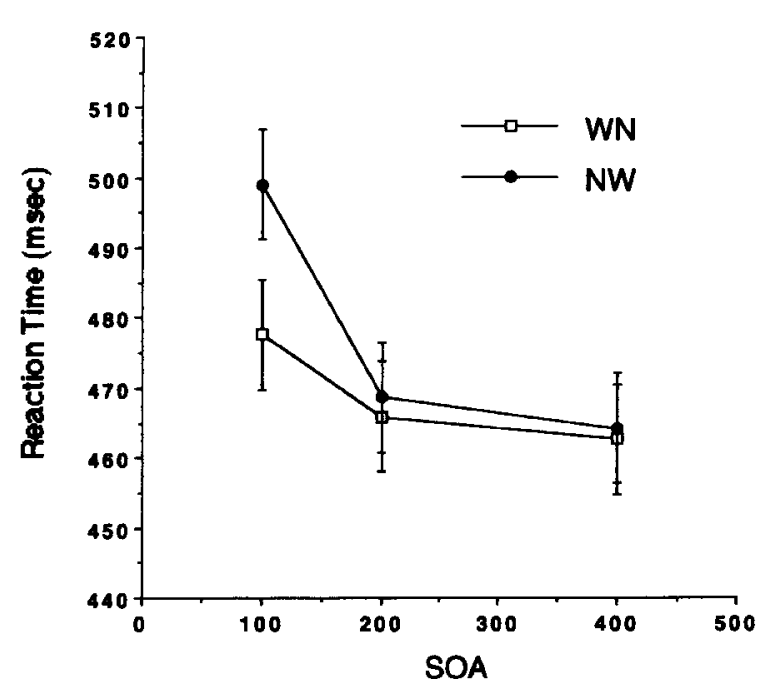

Figure 1. Mean correct reaction time in milliseconds as a function of stimulus onset asynchrony (SOA) and target familiarity in experimental trials. The error bars represent $95 \%$ confidence intervals, as recommended in Loftus and Masson (1994). WN, reference trial in which word was target; $N W$, reference trial in which nonword was target.

were excluded. Approximately $0.24 \%$ of the scores were discarded.

All of the RTs and their associated percentages correct are listed in Table 1. The results from experimental trials are presented graphically in Figure 1 in accordance with Loftus and Masson (1994), with 95\% confidence intervals based on error variance for the familiarity manipulation only. ${ }^{2}$ Independent planned contrasts carried out for the control and experimental RT data at the $100-\mathrm{msec}$ SOA indicated a significant difference in the experimental condition $[F(1,29)=8.45, p=.0069]$ and not in the control condition $[F(1,29)=1.44, p=.24]$. Although the control and the experimental conditions at this SOA appear to differ only by degree (see Table 1), it must be noted that the RT difference in the control condition, in addition to being statistically unreliable, was accompanied by a significant drop in the accuracy for WW as opposed to NN $[F(1,29)=$ $7.69, p=.01]$, whereas in the experimental condition there was no difference in accuracies $[F(1,29)<1]$. This demonstrates that the apparent similarity in trends in the control and experimental conditions may have been due to a speed-accuracy tradeoff in the control conditions.

Following the recommendation of Townsend and Ashby (1983), the data were converted to efficiency scores (RT divided by the proportion correct) in order to allow comparisons among conditions uncontaminated by any existing speed-accuracy tradeoffs. ${ }^{3}$ These scores are also presented in Table 1. Because performance was more efficient with word than with nonword targets in both the 100- and the 200-msec SOA conditions, these SOAs were combined in our planned comparison, which showed a reliable effect $[F(1,29)=7.55, p=.01]$. When the same planned comparison was performed on the control data, there was no difference $(F<1)$.

\section{DISCUSSION}

These results demonstrate that early in the time course of encountering one novel and one familiar item there is a relative benefit for discriminating changes at the location of the familiar item. This finding provides the first evidence that a familiar item in a visual display may rapidly and exogenously attract attention. At later stages (up to $400 \mathrm{msec}$ ), there is no effect of relative familiarity whatsoever. Endogenously generated shifts toward novel items may occur in situations in which information contained within the representations of the items is required by the subject (as in identification and localization; see below), but in this experiment, identity information was not required to perform the motion discrimination task.

These findings support Flowers, Polansky, and Kerl's (1981) attentional explanation for the pattern of performance that they obtained when one of several letter strings was postcued in an identification task. Flowers et al. found that recall of letters in one of three briefly presented strings was hindered if another string in the visual display contained a word, and they hypothesized that the familiar item attracted attention, causing the unfamiliar letter strings to be processed less efficiently. However, because Flowers et al. used postcues and required report of identity information, any effects of familiarity could have been occurring in memory, rather than through the exogenous control of visual attention. Nevertheless, our results indicate that an exogenous shift of visual attention toward the word string may have been responsible for these results, providing a more direct foundation for Flowers et al.'s original explanation.

There are, however, findings and statements in the literature that appear to contradict our conclusion. For example, if familiar items exogenously attract attention, one might expect them to pop out in a visual search task. Neither Flowers and Lohr (1985) nor Reicher et al. (1976) found such an effect when subjects searched for a familiar item in an array of unfamiliar ones. This finding is ambiguous, however, because search rate is determined by both detection of the target (which ought to be affected by the exogenous orienting that we have discovered here) and by rejection of the distracters, which should be faster when they are familiar (Reicher et al., 1976). Thus, in comparisons of searches for words in a background of nonwords with nonwords in a background of words, these two factors will tend to cancel each other out.

In another line of research, Johnston et al. (1990) and Johnston et al. (1993) have stated that novel items attract attention exogenously. In some of their experiments, they found that in arrays of words that were presented to the subject a large number of times, the location of a word that had not been seen before in the context of the experiment was recalled with increased accuracy to the detriment of recalling the location of the familiar words. These experiments failed to meet one of the criteria that we have set forth above - namely, that the reported attribute of the items that is used to index the locus of attention must not be available from the stored representation. When the subject is asked where a word was, by presentation of that word 
alone after the stimulus array, the identity of that word must be matched up with a previously presented identity and attached to a location. This makes the identity, which is contained in the stored representation, an integral part of the localization task.

It must also be noted that Johnston et al.'s $(1990,1993)$ novelty manipulation is quite different from ours. In their experiments, "familiar" items were those that occurred in the context of the experiment much more often than novel ones. Johnston et al. (1993) concluded that "the pattern is dependent not so much on the familiarity of the individual objects composing the visual field as on the unitization of the field as a whole" (p. 152). We were interested in testing whether the existence of stored representations for presented items would influence the degree of activation in the visual buffer. Johnston et al.'s manipulation is orthogonal to this question. Although Johnston et al.'s experiments do not tell us about the differential effects on attention of wellknown and poorly known items in a visual display, they do make a contribution by extending to learned scenes the well-established phenomenon of unexpected items popping out in natural scenes (Loftus \& Mackworth, 1978).

In summary, we have presented two criteria that must be satisfied in order for one to determine whether familiarity (in the sense of an influence from stored representations) exogenously influences the allocation of visual attention: Familiar and unfamiliar items must be presented in the same display at unpredictable locations, and the subject's task must not involve information available from stored representations of the items in the display. When we implemented these criteria, we found that attention had been exogenously drawn to the location of the familiar item. The methodology we developed to produce this demonstration provides a model for the design of future experiments addressing the nature of the knowledge/attention interface.

\section{REFERENCES}

EARHARD, B. (1968). Perception and retention of familiar and unfamiliar materials. Journal of Experimental Psychology, 76, 584-595.

FARAH, M. J. (1990). Visual agnosia: Disorders of object recognition and what they tell us about normal vision. Cambridge, MA: MIT Press.

Flowers, J. H., \& LOHR, D. J. (1985). How does familiarity affect visual search for letter strings? Perception \& Psychophysics, 37, 557-567.

Flowers, J. H., Polansky, M. L., \& KerL, S. (1981). Familiarity, redundancy and the spatial control of visual attention. Journal of Experimental Psychology: Human Perception \& Performance, 7, 157-166.

GERNSBACHER, M. A. (1984). Resolving 20 years of inconsistent interactions between lexical familiarity and orthography, concreteness, and polysemy. Journal of Experimental Psychology: General, 113, 256-281.

Hawkins, H. L., Hillyard, S. A., Luck, S. J., Mouloua, M., DownING, C. J., \& WoODWARD, D. P. (1990). Visual attention modulates signal detectability. Journal of Experimental Psychology: Human Perception \& Performance, 16, 802-811.

Johnston, W. A., Hawley, K. J., Farnham, J. (1993). Novel popout Empirical boundaries and tentative theory. Journal of Experimental Psychology: Human Perception \& Performance, 19, 140-153.

Johnston, W. A., Hawley, K. J., Plewe, S. H., Elliott, J. M. G., \& DEWITT, M. J. (1990). Attention capture by novel stimuli. Journal of Experimental Psychology: General, 119, 397-411.

Klein, R. M., Kingstone, A., \& PonTefract, A. (1992). Orienting of visual attention. In K. Rayner (Ed.), Eye movements and visual cognition: Scene perception and reading (pp. 46-65). New York: SpringerVerlag.
KossLYN, S. M. (1980). Image and mind. Cambridge MA: Harvard University Press.

KRUEGER, L. E. (1970). Search time in a redundant visual display. Journal of Experimental Psychology, 83, 391-399.

LofTUS, G. R., \& MACKWORTH, N. H. (1978). Cognitive determinants of fixation location during picture viewing. Journal of Experimental Psychology: Human Perception \& Performance, 4, 565-572.

LofTUs, G. R., \& MASson, E. J. (1994). A proposed confidence interval for use in within-subject designs. Psychonomic Bulletin \& Review, 1 , 476-490.

McClelland, J. L., \& Rumelhart, D. E. (1981). An interactive activation model of context effects in letter perception: Part I. An account of basic findings. Psychological Review, 88, 375-407

MEwhort, D. J. K., \& Johns, E. E. (1988). Some tests of the interactiveactivation model for word identification. Psychological Research, $\mathbf{5 0}$, 135-147.

Posner, M. I., SNyder, C. R. R., \& Davidson, B. J. (1980). Attention and the detection of signals. Journal of Experimental Psychology: General, 109, 160-174.

Reicher, G. M., SNyder C. R. R., \& RichaRds, J. T. (1976). Familiarity of background characters in visual scanning. Journal of Experimental Psychology: Human Perception \& Performance, 2, 522-530.

Townsend, J. T., \& AshBy, F. G. (1983). Stochastic modelling of elementary psychological processes. New York: Cambridge University Press.

\section{NOTES}

1. The 30th subject's raw data were unavailable for the outlier check, but the uncorrected means were included.

2. Traditional analyses of variance (ANOVAs) were performed on both the control and experimental data. For RTs in the experimental conditions, there was a main effect of the familiarity manipulation $[F(1,29)=$ $5.24, p=.03]$ and a significant effect of $\operatorname{SOA}[F(2,58)=8.09, p=.001]$. The interaction between these two was not significant $[F(2,58)=1.63$, $p=.20]$. In the control conditions, there was a main effect of SOA $[F(2,58)=19.00, p=.001]$, with no effect of familiarity $[F(1,29)=$ $1.72, p=.20]$ and no interaction $(F<1)$. For percent correct data in the experimental condition, there was no effect of target familiarity $[F(1,29)<$ 1], no effect of SOA $[F(2,58)<1]$, and no interaction $[F(2,58)=1.52$, $p=.23]$. In the control condition, there was a main effect of target familiarity $[F(1,29)=7.69, p=.01]$, no effect of SOA $[F(2,58)<1]$, and no interaction $[F(2,58)<1]$.

3. ANOVAs similar to those performed on the RTs and percentage correct (see note 2) were also performed on these scores. For the experimental conditions there was a marginal effect of the familiarity manipulation $[F(1,29)=3.71, p=.06]$ and a significant effect of SOA $[F(2,58)$ $=8.12, p=.0008]$. The interaction between these two was not significant $(F<1)$. In the control conditions, there was a main effect of SOA $[F(1,29)=9.24, p=.0003]$, with $F<1$ for both the familiarity manipulation and the interaction.

APPENDIX

Words and Nonwords Used in the Experiment

\begin{tabular}{llll}
\hline & Words & \multicolumn{2}{c}{ Nonwords } \\
\hline super & chili & uprse & liihc \\
roach & icing & aohcr & ncigi \\
joker & ulcer & reojk & urlce \\
boxer & chore & obxre & hreoc \\
mixer & lousy & xeimr & yuslo \\
stale & prong & tsael & ndgro \\
belch & batch & bclhe & tbcah \\
boost & racer & osotb & rraec \\
rhino & leach & inrho & ehcel \\
chess & cider & shcse & cderi \\
booze & fudge & ezobo & guedf \\
burns & denim & srnbu & nidme \\
\hline
\end{tabular}

(Manuscript received May 10, 1994; revision accepted for publication October 6, 1994.) 